\title{
Polarization dependent loss of arc-induced long-period fibre gratings
}

\author{
Gaspar M. Rego ${ }^{\text {a,b,*, José L. Santos }}{ }^{\text {b,c }}$, Henrique M. Salgado ${ }^{\text {b,d }}$ \\ a Escola Superior de Tecnologia e Gestão, IPVC, Av. do Atlântico, 4900-348 Viana do Castelo, Portugal \\ b UOSE, INESC-Porto, Rua do Campo Alegre 687, 4169-007 Porto, Portugal \\ ${ }^{\mathrm{c}}$ Departamento de Física, FCUP, Rua do Campo Alegre 687, 4169-007 Porto, Portugal \\ ${ }^{\mathrm{d}}$ Departamento de Engenharia Electrotécnica e de Computadores, FEUP, Rua Dr. Roberto Frias, 4200-465 Porto, Portugal
}

Received 25 October 2005; received in revised form 28 December 2005; accepted 28 December 2005

\begin{abstract}
We have investigated the polarization properties of long-period fibre gratings fabricated using the electric arc technique. It was found that the choice of the fabrication parameters (electric current, arc duration and pulling tension) affects the polarization dependent loss of the produced gratings. In particular, a non-monotonic dependence on the external pulling tension was obtained.
\end{abstract}

(C) 2006 Elsevier B.V. All rights reserved.

Keywords: Polarization dependent loss; Fibre gratings; Long-period gratings; Arc-discharges

\section{Introduction}

The equalization of the gain spectra of erbium doped fibre amplifiers (EDFAs) is one of the most important applications of long-period fibre gratings (LPFGs) in optical communications. In such application, the performance of the optical filter is directly related to its polarization properties. Several studies concerned with the polarization dependent loss (PDL) of gratings produced by UV and $\mathrm{CO}_{2}$ laser radiation have been published [1-4]. However, PDL measurements on arc-induced gratings are only briefly described in two papers $[5,6]$. Moreover, the presented results were obtained by considering solely two orthogonal polarizations and, therefore, they correspond somehow to an approximation, since for optical components, such as mechanically induced long-period gratings [7] that possess both PDL and polarization mode dispersion (PMD), the two principal states of polarization corresponding to maximum and minimum power values are not orthogonal [8].

\footnotetext{
* Corresponding author. Tel.: +351 226082 601; fax: +351 226082 699/ 799.

E-mail address: gmrego@fc.up.pt (G.M. Rego).
}

In this paper we discuss the polarization dependent loss of arc-induced gratings under different fabrication parameters.

\section{Fabrication of the gratings}

LPFGs were written in the Corning SMF28 fibre using the electric arc-discharge technique [9]. The fabrication process consists in placing an uncoated fibre between the electrodes of a fusion splice machine (electrodes gap $=$ $0.9-1.0 \mathrm{~mm}$, electrodes apex angle $\left.=20-40^{\circ}\right)$. One end of the fibre is clamped in a fibre holder on top of a motorized translation stage (MTS) controlled with a precision of $0.1 \mu \mathrm{m}$. At the other end a weight is attached to keep the fibre under a constant axial tension (1-40 g). An arc discharge is then produced with an electric current of 8.5$10.0 \mathrm{~mA}$ during $0.5-2.0 \mathrm{~s}$ exposing a short length of the fibre. Afterwards the fibre is moved by the grating period, typically $400-700 \mu \mathrm{m}$. The sequence arc discharge-fibre displacement (computer controlled) is repeated several times (15-100) until a required transmission loss is obtained. The gratings spectra were recorded using an ANDO AQ6315B optical spectrum analyzer (OSA), the illumination being provided by a white light source (WLS) 


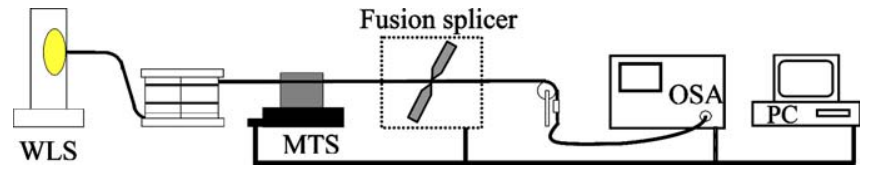

Fig. 1. Experimental setup used for gratings fabrication.

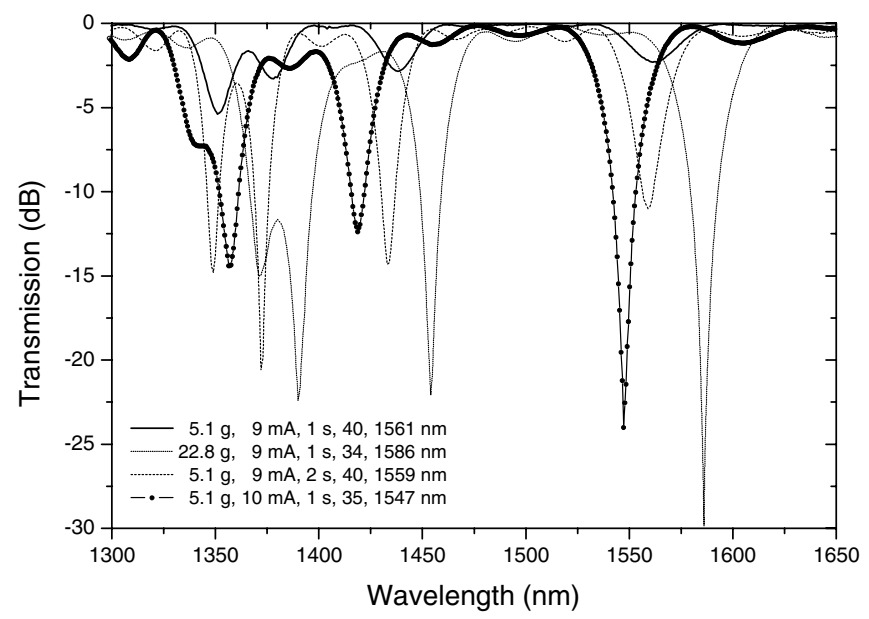

Fig. 2. Influence of the fabrication parameters on the LPFGs spectra.

(Fig. 1). The transmission spectra of several gratings written under different fabrication parameters are shown in Fig. 2. As can be seen, as the external tension, or the electric current, or even the arc duration increases the strength of the gratings also increases. The influence of the fabrication parameters on the polarization properties of the arcinduced gratings is discussed next.

\section{Polarization dependent loss measurements}

The PDL properties of the produced gratings were investigated using the setup shown in Fig. 3. The light from the Photonetics EDFA, was polarized linearly by using a fibre polarizer. The polarization state of the input light is afterwards scanned by the polarization controller, an Agilent 11896A, before going through the LPFG and be detected by the OSA. The PDL values were measured by searching at a particular wavelength in the vicinity of the resonant wavelength, for the maximum and minimum transmitted power. Afterwards, the spectrum of the grating corresponding to the fast and slow axis was registered and the PDL determined by the absolute difference of those spectra. On a second iteration, the whole process was

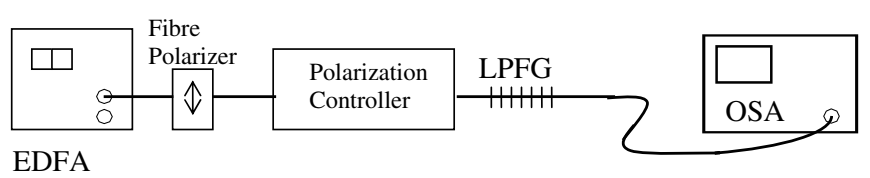

Fig. 3. Experimental setup for PDL measurement.
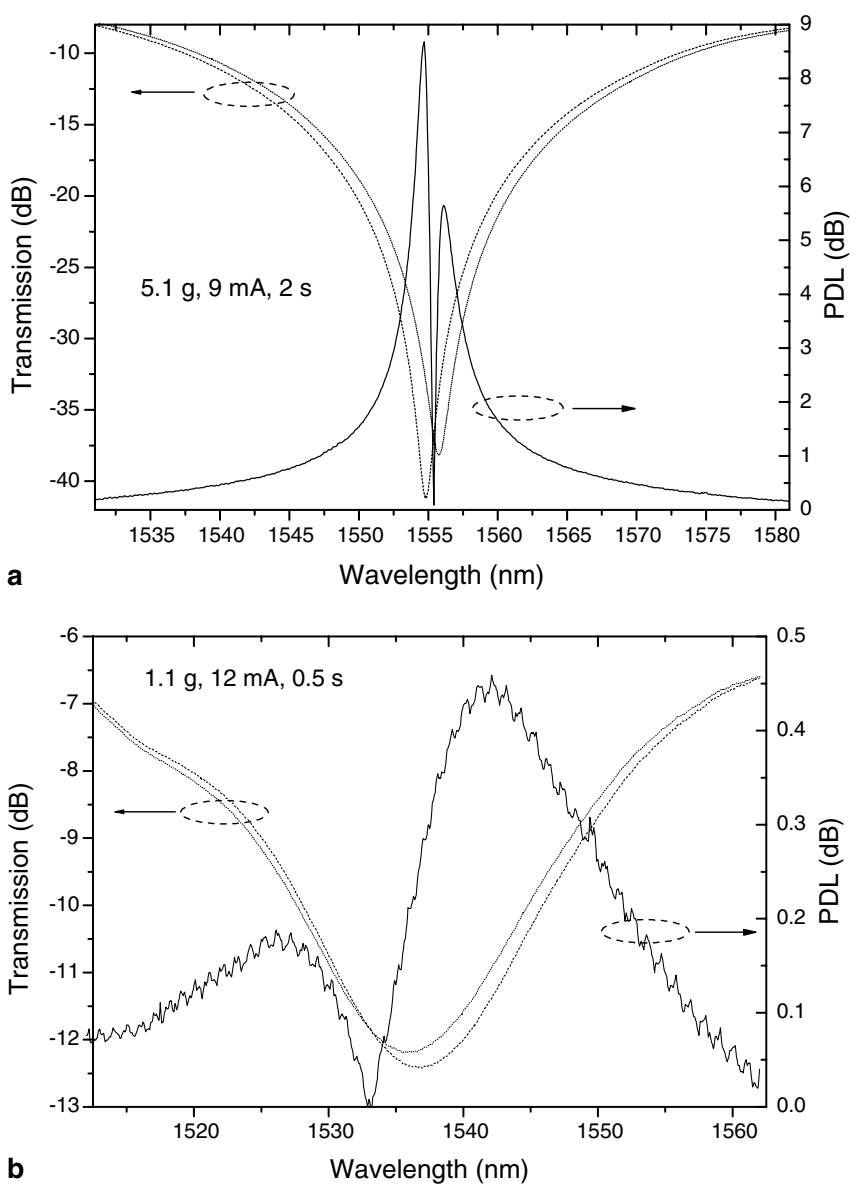

Fig. 4. Two gratings showing the: (a) maximum and (b) minimum PDL values obtained.

repeated at the wavelength where the maximum PDL value occurred. Therefore, although the wavelength dependence of the PDL may comport some error the maximum value of the PDL is fairly accurate. Fig. 4 shows the transmission spectrum of two gratings for the fast and slow axis, and also the two extreme $\mathrm{PDL}_{\max }$ values obtained for these gratings. A maximum value of $8.5 \mathrm{~dB}$ was obtained for a grating written using an arc discharge of $9 \mathrm{~mA}$ during $2 \mathrm{~s}$ whilst applying a tension of $5.1 \mathrm{~g}$ to the fibre. The minimum $\mathrm{PDL}_{\max }$ value was of $0.45 \mathrm{~dB}$ and was obtained for a grating written using an arc discharge of $12 \mathrm{~mA}$ during $0.5 \mathrm{~s}$ whilst applying a tension of $1.1 \mathrm{~g}$ to the fibre. Note that a similar PDL value was also obtained for a grating produced by using an arc-discharge of $10 \mathrm{~mA}$ for $1 \mathrm{~s}$ and the same pulling tension of $1.1 \mathrm{~g}$. Fig. 4 also shows that two gratings with similar wavelength separation $(\sim 1 \mathrm{~nm})$ may exhibit completely different PDL values. Therefore, an important conclusion can be drawn, that is, the PDL values depend not only on the wavelength separation, but also on the gratings strength and bandwidth. Fig. 5 shows the $\mathrm{PDL}_{\max }$ versus the transmission loss for gratings arcinduced in the SMF28 fibre. Note that for the same number of arc discharges employed, gratings written with an external tension of $5.1 \mathrm{~g}$ show different transmission loss values. 


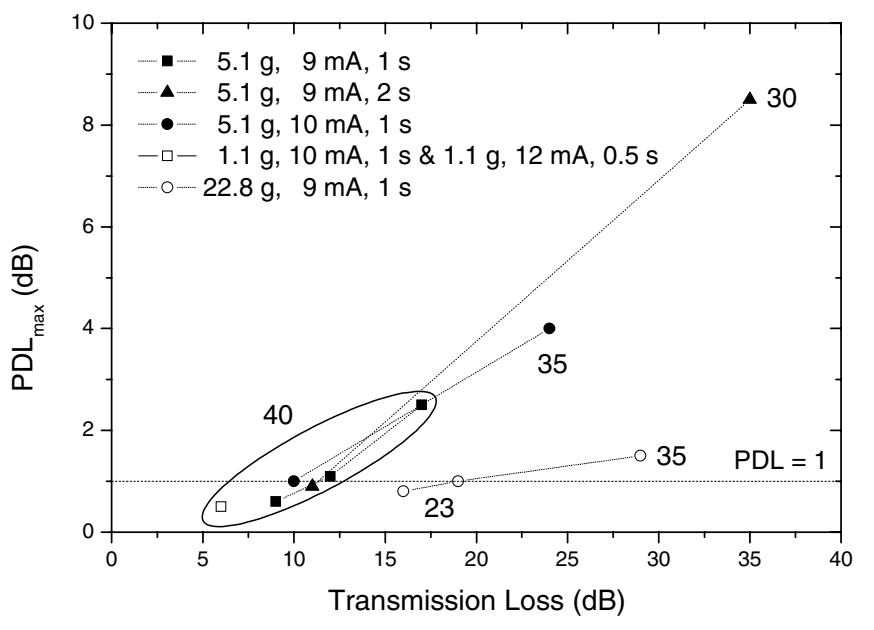

Fig. 5. PDL values as a function of the transmission loss for several gratings written in the Corning SMF28 fibre. The number of arc discharges employed is also indicated in the figure.

The issues related to the reproducibility of the electric arc technique are discussed in Ref. [10]. In any case, it can be concluded that as the transmission loss increases the maximum PDL value also increases and that for the same transmission loss, the use of a higher tension leads to lower PDL values.

To validate the previous results, the PDL of a new set of gratings written in another standard singlemode fibre, from Siecor $(6 \mathrm{~mol} \% \mathrm{Ge})$, was measured by the polarization scanning method [3]. The instruments used were a computer controlled polarization analyzer (HP8509B) and an external tunable laser (HP8167A). For each wavelength, all polarization states in the Poincaré sphere were virtually generated in order to find the minimum and maximum power transmitted through the grating. Fig. 6 shows the transmission spectrum of one grating for the fast and slow axis as determined by the polarization scanning method. The absolute difference of these two curves gives the correspondent PDL values. It was experimentally verified that, for each wavelength, the two principal states of polarization, that corresponds to a maximum and to a minimum, are not orthogonal which confirms that LPFGs possess both PDL and differential group delay (DGD). The PMD is in general determined as an average of the DGD, but in the presence of PDL their relationship is non-trivial [8]. The DGD was software calculated from the wavelength dependence of the Jones matrix eigenvalues and an estimation of its spectral dependence is shown in Fig. 6(b). These results had to be performed in steps of $1 \mathrm{~nm}$ due to a limitation of the equipment used. The maximum PDL values obtained for several gratings arc-induced in the Siecor fibre using different pulling tensions are shown in Fig. 7. In general, these values are slightly higher than the ones presented previously, nevertheless, this figure allows for the same conclusions as the ones drawn from Fig. 5.
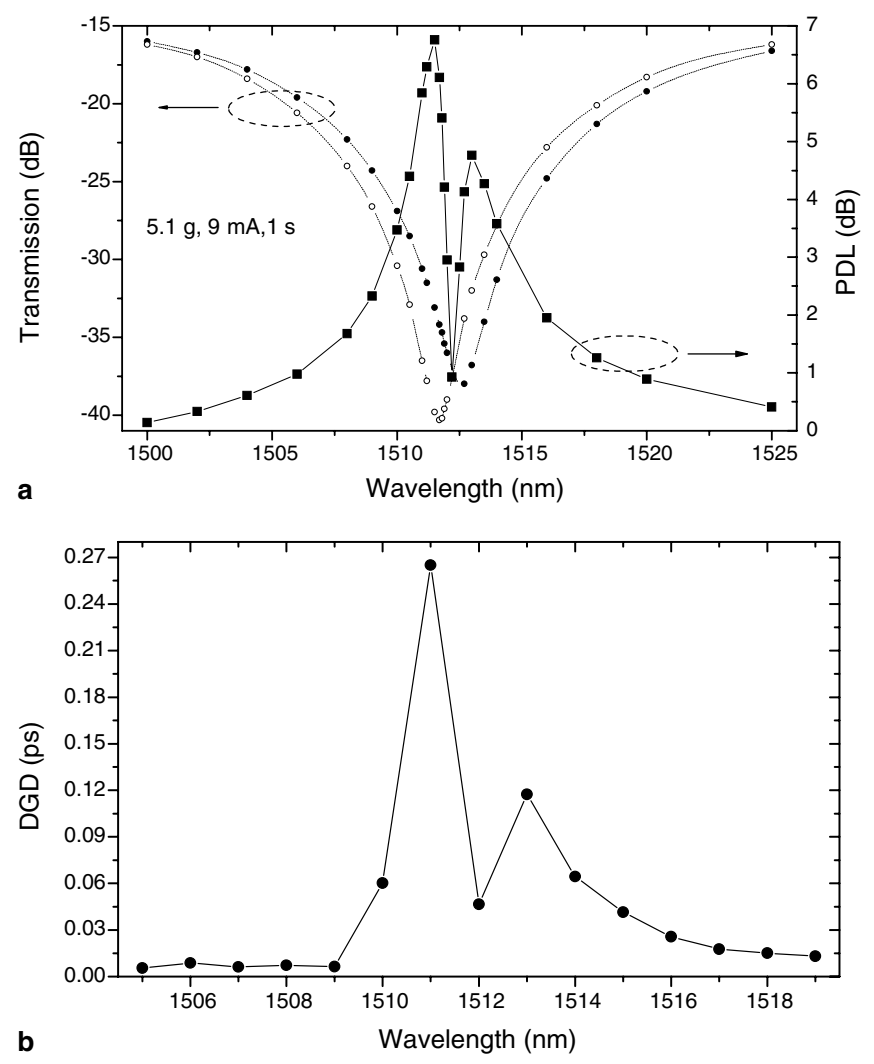

Fig. 6. (a) Transmission spectrum of the grating for two polarizations and the correspondent spectral PDL as obtained in the Poincaré sphere. (b) Spectral DGD of the grating.

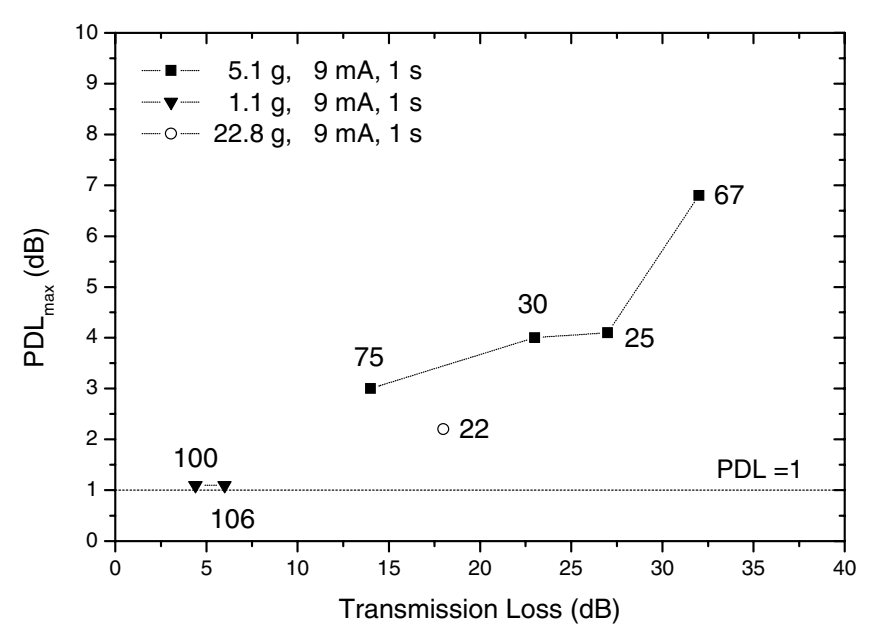

Fig. 7. PDL values as a function of the transmission loss for several gratings written in the Siecor fibre. The number of arc discharges used is also indicated.

\section{Discussion}

In this section we will compare the PDL values obtained experimentally with results published in literature, for gratings produced using different techniques. Fig. 8 summarizes that comparison. In the following discussion of the results, data will appear in the form (Transmission Loss, PDL $_{\max }$ ). 


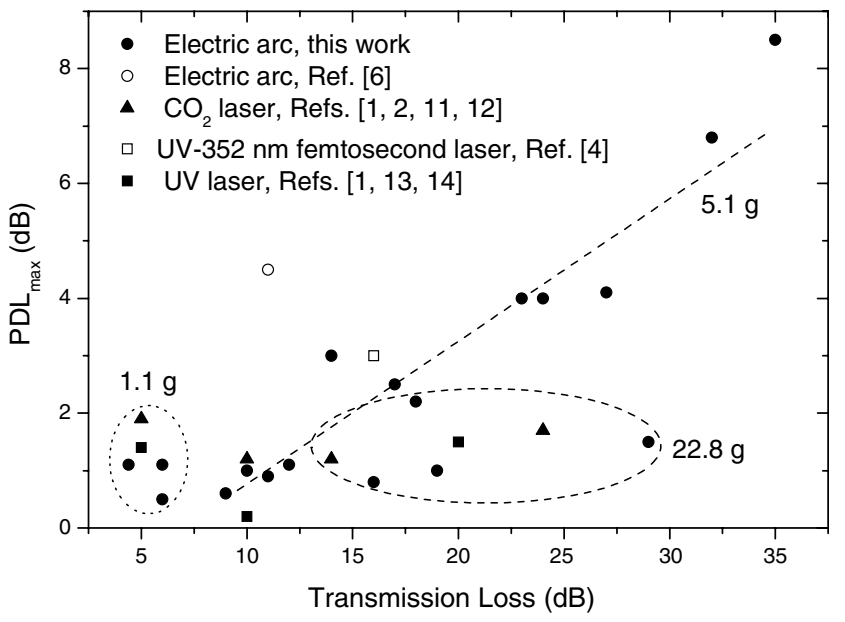

Fig. 8. Comparison of PDL values for gratings produced using different techniques.

As can be seen, the PDL value $(11,4.5)$ obtained by Kim et al. [6] for an arc-induced grating is higher than the ones presented in this work. An even higher value is expected for gratings induced by microbending through arc discharges [5] since it is known that, in general, microbending leads to gratings with large PDL values. In fact, a value as large as $18 \mathrm{~dB}$ was achieved for a mechanically induced grating [7] (not shown in Fig. 8). Also a large value for PDL (16, 8) was obtained by Zhu et al. [3], however, in this case it is not known the technique used to produce the grating. The PDL value $(16,3)$ exhibited by a grating produced by high intensity femtosecond $352 \mathrm{~nm}$ pulses [4] fits well the experimental results obtained for gratings fabricated with a pulling tension of $5.1 \mathrm{~g}$. It is interesting to note that gratings induced by $\mathrm{CO}_{2}$ laser pulses show PDL values between 1 and $2 \mathrm{~dB}$ for a large range of transmission loss values: $(5,1.9)[11] ;(10,1.2)[1] ;(14,1.2)[2] ;(24,1.7)$ [12]. The PDL of the weaker grating is higher than the one obtained for arc-induced gratings produced using a $1.1 \mathrm{~g}$. The PDL of the $10 \mathrm{~dB}$ grating is comparable to gratings produced with $5.1 \mathrm{~g}$ whilst for gratings with greater coupling strengths the PDL compares to the $22.8 \mathrm{~g}$-LPFGs. The PDL of UV-induced gratings $(244-248 \mathrm{~nm})$ ranges from about zero to $1.5 \mathrm{~dB}:(5,1.4)[13] ;(10,0.2)$ [1]; $(20$, 1.5) [14]. These values compares to the ones obtained for arc-induced gratings in case of low (1.1 g) and high pulling tension $(22.8 \mathrm{~g})$. For the intermediate transmission loss, the PDL value is remarkably lower than other obtained for gratings produced by other techniques.

In the case of UV-induced gratings, PDL results from birefringence induced in the fibre core due to one-side exposure. The PDL values are intrinsically low and can be further reduced by a double-side UV exposure [15] or by heating the fibre during gratings inscription [13]. The latter can be achieved by using a heating coil or by a proper choice of the pulses repetition rate. The thermal heat generated will enhance the fibre photosensitivity and will lead to a decrease of the induced birefringence. Single-side exposure and birefringence also induced in the fibre cladding are the reasons pointed by the authors [4] to explain the higher PDL value obtained for a grating induced by femtosecond laser pulses when compared to gratings produced by UV and $\mathrm{CO}_{2}$ laser radiation. As discussed in [1], gratings produced by $\mathrm{CO}_{2}$ laser radiation promotes changes in both the core and the cladding and therefore the PDL values of those gratings are, in principle, higher than for UV gratings. Oh et al. [11] proposed two methods to reduce the gratings PDL: a double-side exposure or rotation of the fibre during exposure to the $\mathrm{CO}_{2}$ laser radiation. As far as arc-induced gratings are concerned, it was recently demonstrated that arc discharges promotes asymmetric stress relaxation in the cladding of optical fibres [16] and therefore, according to [1], arc-induced gratings falls in the same category as the ones produced by $\mathrm{CO}_{2}$ laser pulses. Indeed, both techniques enable the fabrication of gratings showing similar PDL values, considering that the larger pulling tension is used to produce strong arc-induced gratings. It is known that the increase of the pulling tension leads to a reduction of the fibre cross section which in turn enhances the coupling strength and decreases the number of periods required to achieve a particular transmission loss [10]. Simultaneously, it is believed that this geometric effect might contribute to obtain a more homogeneous distribution of birefringence. Finally, it should be stressed that independently of the technique used to fabricate the gratings, their PDL values can be substantially reduced by using a Sagnac loop interferometer [17].

\section{Conclusion}

We have investigated the polarization properties of arcinduced long-period fibre gratings. It was shown that the polarization dependent loss is affected by the values of the fabrication parameters used. In particular, it was found that, for the same strength of the gratings, the use of a higher pulling tension leads to a considerable reduction on the maximum PDL value. A comparison of the PDL values obtained for gratings produced using different techniques was also presented.

\section{Acknowledgement}

G.M. Rego is thankful for the grant conceded by the Program PRODEP III.

\section{References}

[1] B. Bachim, T. Gaylord, Appl. Opt. 42 (2003) 6816.

[2] H.S. Ryu, Y. Park, S.T. Oh, Y.J. Chung, D.Y. Kim, Opt. Lett. 28 (2003) 155.

[3] Y.H. Zhu, E. Simova, P. Berini, C.P. Grover, IEEE Trans. Instrum. Measurem. 49 (2000) 1231.

[4] C. Caucheteur, A. Fotiadi, P. Mégret, S.A. Slattery, D.N. Nikogosyan, IEEE Photon. Technol. Lett. 17 (2005) 2346.

[5] I. Hwang, S. Yun, B. Kim, Opt. Lett. 24 (1999) 1263. 
[6] M. Kim, D. Lee, B. Hong, H. Chung, J. Korean Phys. Soc. 40 (2002) 369.

[7] G. Rego, M. Morais, J.L. Santos, H.M. Salgado, PDL and DGD measurements of mechanically induced long-period fiber gratings, in: London Communications Symposium, University College of London, 2003. Available from: <http://www.ee.ucl.ac.uk/lcs/papers2003/ 101.pdf $>$.

[8] B. Huttner, C. Geiser, N. Gisin, J. Select Top. Quantum Electron. 6 (2000) 317.

[9] G. Rego, O. Okhotnikov, E.M. Dianov, V. Sulimov, J. Lightwave Technol. 19 (2001) 1574.

[10] G. Rego, P.S. Marques, H.M. Salgado, J.L. Santos, Fiber Integrated Opt. 24 (2005) 245.
[11] S.T. Oh, W.T. Han, U.C. Paek, Y.J. Chung, Microwave Opt Technol. Lett. 41 (2004) 188

[12] D.D. Davis, T.K. Gaylord, E.N. Glytsis, S.C. Mettler, Electron. Lett. 34 (1998) 1416.

[13] S.T. Oh, W.T. Han, U.C. Paek, Y. Chung, Opt. Exp. 11 (2003) 3087.

[14] B.H. Lee, J. Cheong, U.C. Paek, Opt. Lett. 27 (2002) 1096.

[15] A.M. Vengsarkar, Q. Zhong, D. Inniss, W.A. Reed, P.J. Lemaire, S.G. Kosinski, Opt. Lett. 19 (1994) 1260.

[16] F. Dürr, G. Rego, P.V.S. Marques, S.L. Semjonov, E.M. Dianov, H.G. Limberger, R.P. Salathé, J. Lightwave Technol. 23 (2005) 3947.

[17] C.-S. Kim, B. Choi, J.S. Nelson, Q. Li, P.Z. Dashti, H.P. Lee, Opt. Lett. 30 (2005) 20. 